\title{
SYNTHESIS OF NOVEL TRYPTOPHAN DERIVATIVES OF POTENTIAL BIOLOGICAL ACTIVITY
}

\author{
RAFAT M. MOHAREB ${ }^{A, B *}$, NADIA A. LOUCAC , GAMAL A. ELMEGEEDC AND HANAA Y. HANAC \\ ${ }^{a}$ Departament of Organic Chemistry, Faculty of Pharmacy, October University for Modem Sciences and Arts (MSA), El-Wahaat Road, October City, Egypt \\ ${ }^{b}$ Chemistry Department, Faculty of Science, Cairo University, Giza, Egypt \\ ${ }^{c}$ Hormone Department, National Research Centre, Dokki, Giza, Egypt \\ (Received: May 27, 2008 -Accepted: December 12, 2008)
}

\begin{abstract}
Tryptophan methyl ester $\mathbf{2}$ reacts with ethyl cyanoacetate to form acetonitrilocarbonyltryptophan methylester $\mathbf{3}$. The latter reacts with cyanomethylene reagents, hydrazines, cyanomethylenes and sulfur to form the corresponding $\alpha$-pyrido-3-indolopropanoate derivatives $\mathbf{6 a}, \mathbf{b}$, pyrazolyltryptophan methyl ester derivatives $\mathbf{8 a}, \mathbf{b}$ and thiophenotryptophan methyl ester derivatives $\mathbf{1 0 a}, \mathbf{b}$, respectively. Also compound $\mathbf{3}$ reacts with benzaldehyde to give the condensated product $\mathbf{1 2}$. The reactivity of the latter product towards chemical reagents was studied to form pyridine, pyrazole and isoxazole derivatives.
\end{abstract}

Keywords: Teyptophane, pyrazd, pyridine, 1, 3 - oxazine.<smiles>CC(=O)C(N)Cc1c[nH]c2ccccc12</smiles>

INTRODUCTION

The development of potential therapeutic agents based on the structure of peptides has stimulated an interest in the design and synthesis of unnatural amino acids [1-3]. Thus, numerous chemical transformations of amino acids have been reported [4]. Indole and it's derivatives have been a topic of research interest for over a century $[5,6]$. This, is in part, due to the fact that indole moieties are found in a variety of naturally occurring compounds that exhibit various physiological properties $[7,8]$. Tryptophan is one of the most important amino acids which containing indole moiety. However, to our knowledge, few investigations have been reported concerning its uses in the formation of cyclic tryptophan derivatives [9-12]. The reaction of tryptophan with cyanoacetates is known to produce cyanoamides in the aim of forming photo-active crosslinking bioprobes $[13,14]$. In view of these observations and in continuation with studies involving the synthesis of unusual hormones and amino acids derivatives [15-17], we have used in this study, $L$-tryptophan is used to form new heterocyclic compounds of potential biological activities.

\section{RESULTS AND DISCUSSION}

The reaction of tryptophan (1) with methanol in acetyl chloride solution afforded the corresponding tryptophan methyl ester 2 (Scheme 1). Compound 2 reacted with an equimolar amount of ethyl cyanoacetate in 1,4-dioxan under reflux to give the methyl $\alpha$-imino(acetonitrilocarbamido)-3-indolopropanoate (3) via elimination of ethanol. The mass spectrum of compound $\mathbf{3}$ showed molecular ion peak at $\mathrm{m} / \mathrm{z} 286(25 \%)$. The IR spectrum of compound $\mathbf{3}$ revealed the presence of NH stretching at $v 3400-3360 \mathrm{~cm}^{-1}$, one $\mathrm{CN}$ group stretching at $v 2220 \mathrm{~cm}^{-1}$ and two $\mathrm{C}=\mathrm{O}$ groups stretching at $v 1730$ and $1680 \mathrm{~cm}^{-1}$. Also the ${ }^{1} \mathrm{H}$ NMR spectrum of compound $\mathbf{3}$ showed two $\mathrm{D}_{2} \mathrm{O}$-exchangeable singlets at $\delta 8.65$ and 8.76 for the two $\mathrm{NH}$ groups ( $c f$. experimental section). Moreover, the ${ }^{13} \mathrm{C}$ NMR spectrum showed $\delta 22.6\left(\mathrm{CH}_{3}\right), 28.9,44.2\left(2 \mathrm{CH}_{2}\right), 50.2(\mathrm{CH})$, 115.1, 116.7, 118.4, 120.4, 121.4, 122.6, 142.6 (pyrole, benzene C), 172.6, $174.8(2 \mathrm{C}=\mathrm{O})$.
Synthesis of compound $\mathbf{3}$

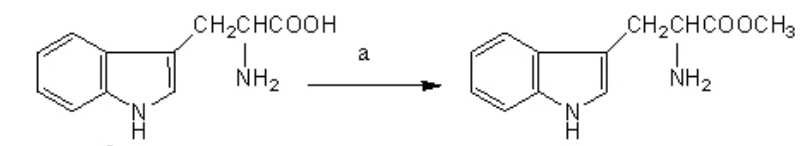<smiles>CC(=O)C(Cc1c[nH]c2ccccc12)NC(=O)CC#N</smiles>

3
1

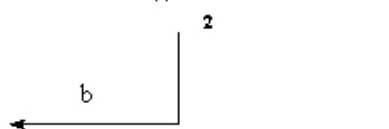

Reagents and conditions: (a) $\mathrm{CH}_{3} \mathrm{COCl}, \mathrm{CH}_{3} \mathrm{OH}$; (b) $\mathrm{NCCH}_{2} \mathrm{COOC}_{2} \mathrm{H}_{5}$, he at

To improve the pharmaceutical properties of the tryptophan molecule, we have outlined the synthesis of several indole derivatives containing heterocyclic moiety. Therapeutic agents containing pyridine, thiophene, and pyrazole moieties have attracted the attention of researchers in pharmaceutical chemistry; these heterocycles have been found to show various biological activities $[18,19]$. Compound 3 seemed to be an interest candidate for further chemical transformations to form indole derivatives with potential biological activity. The reactivity of compound $\mathbf{3}$ towards the reaction with active methylene reagents was investigated. Thus, compound $\mathbf{3}$ reacted with equimolar amount of either malononitrile (4a) or ethyl cyanoacetate (4b) in ethanolic triethylamine solution gave the $\alpha$-pyrido-3-indolopropanoate derivatives $\mathbf{6 a}, \mathbf{b}$, respectively. Formation of the latter products took place via the intermediate formation of $\mathbf{5 a}, \mathbf{b}$ which underwent ready intramolecular cyclization to give compounds $\mathbf{6 a}, \mathbf{b}$ (Scheme 2). The IR spectrum of each of $\mathbf{6 a}$ and $\mathbf{6 b}$ showed two $\mathrm{CN}$ groups stretching at $v 2200$ and $2225 \mathrm{~cm}^{-1}$, respectively. The ${ }^{1} \mathrm{H}$ NMR of compound 6a showed a singlet at $\delta 4.62\left(4 \mathrm{H}, \mathrm{D}_{2} \mathrm{O}\right.$-exchangeable) for the two $\mathrm{NH}_{2}$ groups while that of compound $\mathbf{6 b}$ showed a singlet at $\delta 4.41\left(2 \mathrm{H}, \mathrm{D}_{2} \mathrm{O}\right.$-exchangeable) for one $\mathrm{NH}_{2}$ group and a singlet at $\delta 10.05$ for the $\mathrm{OH}$ group. Moreover, each of compounds $6 \mathbf{a}, \mathbf{b}$ showed a singlet at $\delta 6.23$ and 6.34 , respectively for the pyridine ring proton at $\mathrm{C}-3$ ( $c f$. experimental section).

The study also extended to the reactivity of compound $\mathbf{3}$ towards nitrogen nucleophilic reagents. Thus, compound $\mathbf{3}$ reacted with equimolar amounts of either hydrazine hydrate or phenylhydrazine in refluxing ethanol containing a catalytic amount of triethylamine to give the corresponding pyrazolyltryptophan methyl ester derivatives $\mathbf{8 a}, \mathbf{b}$. The reaction took place via a simple ad- 
dition of hydrazine hydrate or phenylhydrazine to the $\mathrm{CN}$ group in compound $\mathbf{3}$ to give the intermediates $\mathbf{7 a}, \mathbf{b}$ which readily afforded compounds $\mathbf{8 a}, \mathbf{b}$ via water elimination (Scheme 2). Moreover, compound 3 reacted with hydroxylamine hydrochloride in cold ethanolic sodium acetate solution to give the corresponding oxadiazinomethylenoindole derivative $\mathbf{9}$ (Scheme 2). Structures of compounds $\mathbf{8 a}, \mathbf{b}$ and $\mathbf{9}$ were supported by their compatible analytical and spectral data ( $c f$. experimental section).

Synthe sis of compounds $6 \mathrm{a}, \mathrm{b}$ to 9 :

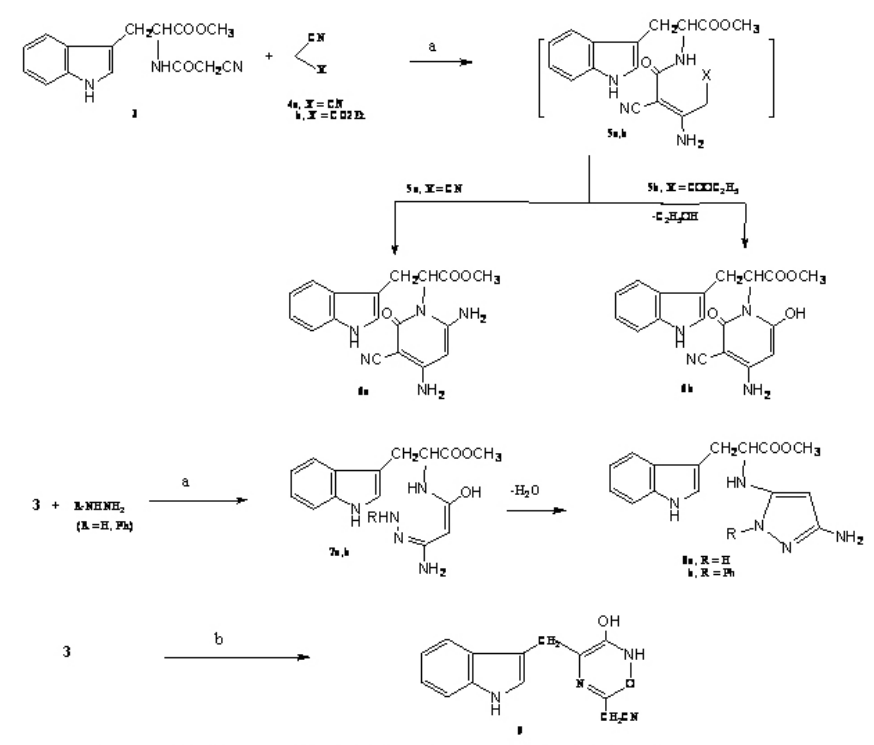

Reagents and conditions: (a) $\mathrm{E} t \mathrm{OH} / \mathrm{E} t 3 \mathrm{~N}$, heat; (b) $\mathrm{NH}_{2} \mathrm{OH}, \mathrm{E}+\mathrm{OH} / \mathrm{AcON}$ a heat

Scheme (2)

Compound 3 reacted with equimolar amounts of either malononitrile $\mathbf{4 a}$ or ethyl cyanoacetate $\mathbf{4 b}$ and elemental sulfur in ethanolic triethylamine solution under reflux to afford the corresponding thiophenotryptophan methyl ester derivatives 10a,b (Scheme 3). The IR spectrum of compound 10a revealed the presence of two $\mathrm{CN}$ groups stretching at $v 2210$ and $2225 \mathrm{~cm}^{-1}$ and the ${ }^{1} \mathrm{H}$ NMR spectrum revealed the presence of $\mathrm{D}_{2} \mathrm{O}$-exchangeable singlet $(2 \mathrm{H})$ at $\delta 4.58$ for the $\mathrm{NH}_{2}$ group and two $\mathrm{D}_{2} \mathrm{O}$-exchangeable singlets at $\delta 10.78(1 \mathrm{H})$ and $\delta 11.94$ $(1 \mathrm{H})$ for the two $\mathrm{NH}$ groups. On the other hand, the IR spectrum of $\mathbf{1 0 b}$ showed the presence of one $\mathrm{CN}$ group stretching at $v 2200 \mathrm{~cm}^{-1}$ and its ${ }^{1} \mathrm{H}$ NMR spectrum showed a triplet at $\delta 1.23$ beside a quartet at $\delta 4.24$ due to the ethyl ester group, in addition to the $\mathrm{NH}_{2}$ and the two $\mathrm{NH}$ singlet signals at $\delta 6.50,10.71$ and 10.88 , respectively. The cyanomethylene moiety of compound 3 coupled easily with benzenediazonium chloride in cold ethanolic sodium acetate solution to give the phenylhydrazo derivative $\mathbf{1 1}$ based on ${ }^{1} \mathrm{H}$ NMR spectrum which showed, beside the expected chemical shifts, three NH singlets at $\delta 9.25$ and 9.97-9.99 ( $\mathrm{D}_{2} \mathrm{O}$-exchangeable) (Scheme 3).
Synthesis of compounds $10 \mathbf{a}, \mathbf{b}$ and $\mathbf{l l}$

$$
\text { R+ }
$$

The reaction of compound 3 with equimolar amount of benzaldehyde in ethanolic triethylamine solution under reflux afforded the condensated product benzalacetonitrilocarbonyltryptophan methyl ester derivative 12 (Scheme 4). The reactivity of the $\alpha, \beta$-unsaturated nitrile moiety of compound $\mathbf{1 2}$ towards the reaction with various chemical reagents was investigated. Thus, 12 reacted with either malononitrile $4 \mathbf{a}$ or ethyl cyanoacetate $\mathbf{4 b}$ in refluxing ethanolic triethylamine solution to give the methyl $\alpha$-pyrido-1-yl-3-indolopropanoate derivatives $\mathbf{1 4 a}, \mathbf{b}$, respectively via the intermediacy of $\mathbf{1 3 a}, \mathbf{b}$ (Scheme 4). The IR spectrum of compound 14a revealed the presence of two $\mathrm{CN}$ groups at $v$ 2220 and $2215 \mathrm{~cm}^{-1}$ and the ${ }^{1} \mathrm{H}$ NMR spectrum of compound 14a showed two $\mathrm{D}_{2} \mathrm{O}$-exchangeable singlets at $\delta 4.46(2 \mathrm{H})$ and $\delta 11.02(1 \mathrm{H})$ corresponding to the $\mathrm{NH}_{2}$ and $\mathrm{NH}$ groups, respectively. The mass spectrum of compound $\mathbf{1 4 b}$ showed a molecular ion peak at $\mathrm{m} / \mathrm{z} 486(35 \%)$ and the ${ }^{1} \mathrm{H}$ NMR spectrum of $14 \mathrm{~b}$ revealed a triplet at $\delta 1.33$ and a quartet at $\delta 4.32$ which is characteristic to ethyl ester group ( $c f$. experimental section).

Synthesis of compounds $\mathbf{1 2}-14 \mathbf{a}, \mathbf{b}$

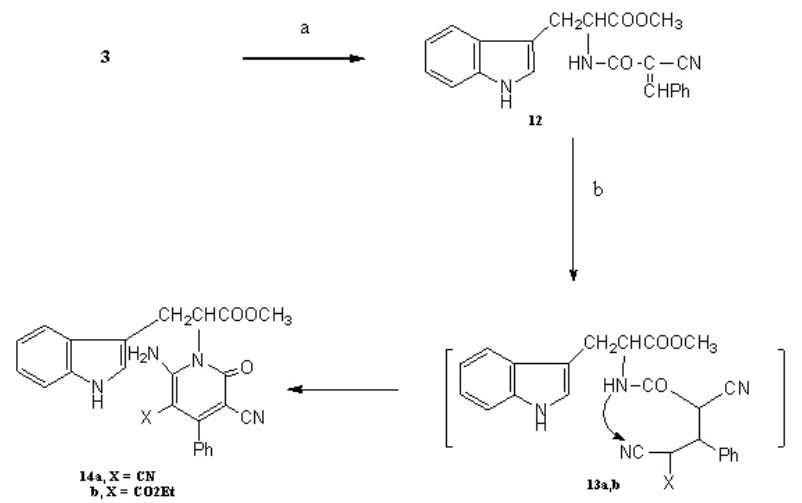

Reagents and conditions: (a) $\mathrm{PhCHO}, \mathrm{EtOH} / \mathrm{Et} 3 \mathrm{~N}$, heat; (b) $\mathrm{NCCH}_{2} \mathrm{COOC}_{2} \mathrm{H}_{5}$ or $\mathrm{NCCH}_{2} \mathrm{CN}$ $\mathrm{EtOH}, \mathrm{Et}_{3} \mathrm{~N}$

\section{Scheme (4)}

The reactivity of compound $\mathbf{1 2}$ towards the reaction with nucleophilic reagents was investigated. Thus, the reaction of $\mathbf{1 2}$ with equimolar amounts of either hydrazine hydrate or phenylhydrazine in ethanolic triethylamine solution under reflux afforded the corresponding carbonylpyrazolotryptophan methyl ester derivatives $\mathbf{1 5 a}, \mathbf{b}$, respectively (Scheme 5 ). Similarly compound $\mathbf{1 2}$ reacted with hydroxylamine hydrochloride in ethanol containing sodium acetate solution to afford the isooxazolotryptophan methyl ester derivative $\mathbf{1 6}$ (Scheme 5 ). The chemical structures of compounds $\mathbf{1 5 a}, \mathbf{b}$ and $\mathbf{1 6}$ were confirmed via the analytical and spectral data ( $c f$. experimental section). 


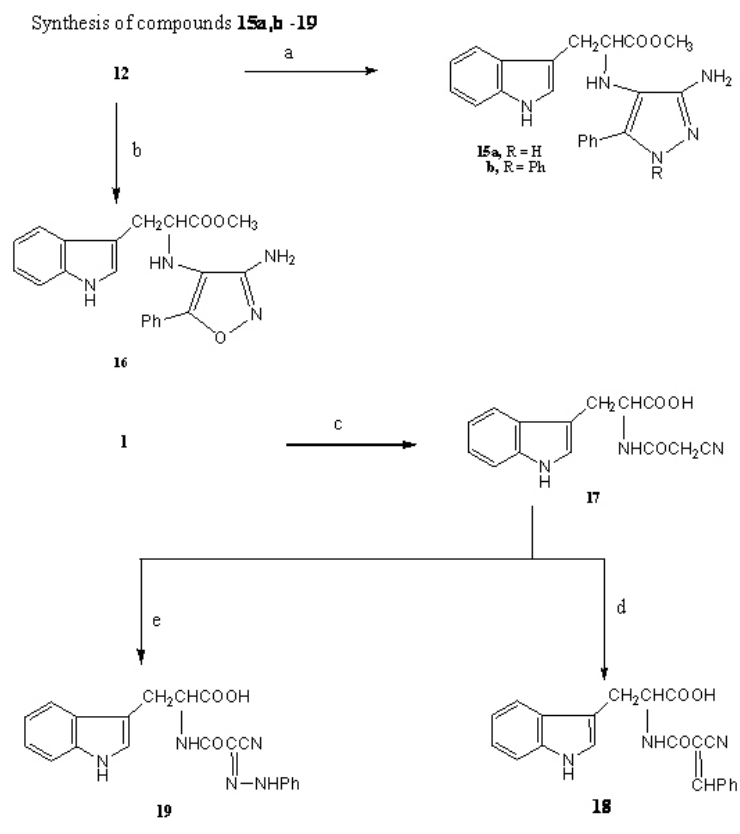

Reagents and conditions: (a) $\mathrm{NH}_{2} \mathrm{NH}_{2}$ or $\mathrm{PhNHNH}_{2}, \mathrm{EtOH} / \mathrm{Et} \mathrm{t}_{3} \mathrm{~N}$; (b) $\mathrm{NH}_{2} \mathrm{OHHCl}, \mathrm{EtOH} / \mathrm{AcONa}$; (c) $\mathrm{NCCH}_{2} \mathrm{COOC}_{2} \mathrm{H}_{5}, \mathrm{DMF}$, heat; (d) $\mathrm{PhCHO}, \mathrm{EtOH}^{2} \mathrm{Et}_{3} \mathrm{~N}$, heat; (e) $\mathrm{PhN}_{2}{ }^{+} \mathrm{Cl}$, $\mathrm{EtOH}, \mathrm{AcONa}$

Scheme (5)

Tryptophan (1) reacted with an equimolar amount of ethyl cyanoacetate in refluxing dimethylformamide solution to give the acetonitrilocarbonyl tryptophan 17 (Scheme 5). The IR spectrum of compound 17 revealed the presence of two $\mathrm{NH}$ groups stretching at $v 3430-3385 \mathrm{~cm}^{-1}$, and one $\mathrm{CN}$ group stretching at $v 2220 \mathrm{~cm}^{-1}$ in addition to two $\mathrm{C}=\mathrm{O}$ groups stretching at $v 1705$ and $1680 \mathrm{~cm}^{-1}$. Also the ${ }^{1} \mathrm{H}$ NMR spectrum of compound 17 showed two singlets at $\delta 8.75$ and $8.82\left(\mathrm{D}_{2} \mathrm{O}\right.$-exchangeable) for the two $\mathrm{NH}$ groups and a singlet at $\delta 11.15$ for the $\mathrm{OH}$ group ( $c f$. experimental section). Compound 17 reacted with an equimolar amount of benzaldehyde in ethanol/piperidine solution under reflux to afford the condensed product benzaloacetonitrilocarbonyl-tryptophan $\mathbf{1 8}$ (Scheme 5). Moreover, compound 17 easily coupled with benzenediazonium chloride in cold ethanolic sodium acetate solution to afford the phenylhydrazo derivative 19 (Scheme 5), based on ${ }^{1} \mathrm{H}$ NMR spectrum which showed, beside the expected chemical shifts, three NH singlets at $\delta 8.51$ and 9.32-9.36 ( $\mathrm{D}_{2} \mathrm{O}$-exchangeable). The structures of the latter products 18 and $\mathbf{1 9}$ were confirmed based on their compatible elemental and spectral analyses ( $c f$. experimental section).

\section{EXPERIMENTAL SECTION}

The starting pure powder of $L$-tryptophan was purchased from Sigma Company, USA. The appropriate precautions in handling moisture sensitive compounds were undertaken. All melting points of the newly synthesized compounds were measured using an electrothermal capillary melting point apparatus and are uncorrected. The IR spectra are expressed in $\mathrm{cm}^{-1}$ and recorded in $\mathrm{KBr}$ pellets on a Pa-9721 IR spectrometer (Shimadzu, Japan). ${ }^{1} \mathrm{H}-$ and ${ }^{13} \mathrm{C}$ NMR spectra were obtained on a Varian EM-390 90 MHz spectrometer in DMSO- $\mathrm{d}_{6}$ as solvent, using TMS as internal reference and chemical shifts $(\delta)$ are expressed in ppm. Mass spectra were recorded on a GCMS-QP 1000 Ex spectra mass spectrometer operating at $70 \mathrm{eV}$ Equipment (Germany). Elemental analyses were preformed with all final compounds by Microanalytical Data Unit at The National Research Centre, Giza, Egypt. The reactions were followed using TLC analyses which were performed using Merck 60 F254 aluminum sheets and visualized by UV light $(254 \mathrm{~nm})$. Although tryptophan methyl ester is commercially available in the hydrochloride form we preferred to syntheses the native methyl ester via the procedure indicated below.
Methyl $\alpha$-amino-3-indolopropanoate (2). To a solution of tryptophan 1 $(10.2 \mathrm{~g}, 0.05 \mathrm{~mol})$ in methanol $(5 \mathrm{~mL})$ equivalent amount of acetylchloride $(3.77 \mathrm{~mL}, 0.05 \mathrm{~mol})$ in methanol $(30 \mathrm{~mL})$ was added drop wise with stirring at $0^{\circ} \mathrm{C}$. The reaction mixture was stirred for $24 \mathrm{~h}$, and then evaporated under vacuum. The formed solid product neutralized by sodium carbonate solution (1 $\mathrm{N})$, extracted by chloroform and dried by anhydrous sodium sulfate. The solid product formed after evaporation of the solvent, filtered off, dried and crystallized from methanol. White crystals, yield $55 \%(6.0 \mathrm{~g})$, mp $152-153^{\circ} \mathrm{C}$. IR $(v /$ $\left.\mathrm{cm}^{-1}\right)$ : 3450-3235 (NH, $\left.\mathrm{NH}\right), 3050\left(\mathrm{CH}\right.$-aromatic) $2985,2865\left(\mathrm{CH}_{3}, \mathrm{CH}_{2}\right) 1730$ $(\mathrm{C}=\mathrm{O}), 1620(\mathrm{C}=\mathrm{C}) .{ }^{1} \mathrm{H}$ NMR $(\delta \mathrm{ppm}): 3.35\left(\mathrm{~s}, 3 \mathrm{H}, \mathrm{CH}_{3}\right), 3.43\left(\mathrm{~m}, 2 \mathrm{H}, \mathrm{CH}_{2}\right)$, $3.75(\mathrm{~m}, 1 \mathrm{H}, \mathrm{CH}), 6.57\left(\mathrm{~s}, 2 \mathrm{H}, \mathrm{NH}_{2}, \mathrm{D}_{2} \mathrm{O}\right.$-exchangeable), $6.64(\mathrm{~s}, 1 \mathrm{H}, \mathrm{CH})$, $7.51\left(\mathrm{~m}, 4 \mathrm{H}, \mathrm{C}_{6} \mathrm{H}_{4}\right), 8.92\left(\mathrm{~s}, 1 \mathrm{H}, \mathrm{NH}, \mathrm{D}_{2} \mathrm{O}\right.$-exchangeable). $\mathrm{MS}(\mathrm{m} / \mathrm{z}, \%): 218$ $\left(\mathrm{M}^{+}, 45 \%\right)$. Anal. Calcd. for $\mathrm{C}_{12} \mathrm{H}_{14} \mathrm{~N}_{2} \mathrm{O}_{2}$ (218.26): $\mathrm{C}, 66.03 ; \mathrm{H}, 6.46 ; \mathrm{N}, 12.83$. Found: $\mathrm{C}, 65.79 ; \mathrm{H}, 6.25 ; \mathrm{N}, 12.52$.

Methyl $\alpha$-imino(acetonitrilocarbamido)-3-indolopropanoate (3). To a solution of $2(1.09 \mathrm{~g}, 0.005 \mathrm{~mol})$ in 1,4-dioxan $(30 \mathrm{~mL})$, equivalent amount of ethyl cyanoacetate $4 \mathbf{b}(0.56 \mathrm{~g}, 0.005 \mathrm{~mol})$ was added. The reaction mixture was heated under reflux for $3 \mathrm{~h}$. The solid product formed by evaporation under vacuum was filtered off, dried and crystallized. White crystals from dioxane, yield $75 \%(1.07 \mathrm{~g}), \mathrm{mp} 128-130^{\circ} \mathrm{C}$. IR $\left(v / \mathrm{cm}^{-1}\right): 3400-3360(2 \mathrm{NH}), 3050$ (CH-aromatic), 2985, $2659\left(\mathrm{CH}_{3}, \mathrm{CH}_{2}\right), 2220(\mathrm{CN}), 1730,1680(2 \mathrm{C}=\mathrm{O}), 1620$ $(\mathrm{C}=\mathrm{C}) .{ }^{1} \mathrm{H}$ NMR $(\delta \mathrm{ppm}): 3.38\left(\mathrm{~s}, 3 \mathrm{H}, \mathrm{CH}_{3}\right), 3.45\left(\mathrm{~m}, 2 \mathrm{H}, \mathrm{CH}_{2}\right), 3.48(\mathrm{~s}, 2 \mathrm{H}$, $\left.\mathrm{CH}_{2} \mathrm{CN}\right), 3.78(\mathrm{~m}, 1 \mathrm{H}, \mathrm{CH}), 6.95(\mathrm{~s}, 1 \mathrm{H}, \mathrm{CH}), 7.01-7.49\left(\mathrm{~m}, 4 \mathrm{H}, \mathrm{C}_{6} \mathrm{H}_{4}\right), 8.65$, $8.76\left(2 \mathrm{~s}, 2 \mathrm{H}, 2 \mathrm{NH}, \mathrm{D}_{2} \mathrm{O}\right.$-exchangeable). ${ }^{13} \mathrm{C}$ NMR $(\delta): 22.6\left(\mathrm{CH}_{3}\right), 28.9,44.2$ $\left(2 \mathrm{CH}_{2}\right), 50.2(\mathrm{CH}), 115.1,116.7,118.4,120.4,121.4,122.6,142.6$ (pyrole, benzene C), 172.6, $174.8(2 \mathrm{C}=\mathrm{O})$. MS (m/z, \%): $\left.286\left(\mathrm{M}^{+}+1\right), 25 \%\right), 260\left(\mathrm{M}^{+}\right.$. $\mathrm{CN}, 25 \%), 203\left(\mathrm{M}^{+}-\mathrm{NHCOCH}_{2} \mathrm{CN}, 23 \%\right), 130$ (indolomethylene fragment, $\mathrm{C}_{9} \mathrm{H}_{8} \mathrm{~N}, 100 \%$ ). Anal. Calcd. for $\mathrm{C}_{15} \mathrm{H}_{15} \mathrm{~N}_{3} \mathrm{O}_{3}(285.30)$ : C, 63.15; H, 5.30; N, 14.73. Found: $\mathrm{C}, 63.42 ; \mathrm{H}, 5.37 ; \mathrm{N}, 14.39$.

Methyl $\alpha$-(2,4-diamino-5-cyano-6-oxopyrido-1-yl)-3-indolopropanoate (6a), Methyl $\alpha$-(4-Amino-5-cyano-2-hydroxy-6-oxopyido-1-yl) -3-indolopropanoate (6b)

\section{General Procedure}

To a solution of Compound $3(1.42 \mathrm{~g}, 0.005 \mathrm{~mol})$ in ethanol $(30 \mathrm{~mL})$, containing a catalytic amount of triethylamine $(1 \mathrm{~mL}, 0.01 \mathrm{~mol})$, equivalent amount of either malononitrile $4 \mathrm{a}(0.33 \mathrm{~g}, 0.005 \mathrm{~mol})$ or ethyl cyanoacetate $4 \mathrm{~b}$ $(0.56 \mathrm{~g}, 0.005 \mathrm{~mol})$ was added. The reaction mixture in each case was heated under reflux for $3 \mathrm{~h}$ and then was cooled at room temperature, poured over ice/ water mixture and neutralized with dilute hydrochloric acid. The formed solid product, in each case filtered off, dried and crystallized from the appropriate solvent.

Compound 6a: Yellow crystals, from $\mathrm{MeOH}$, yield $77 \%$ (1.35g), mp 150-152 ${ }^{\circ} \mathrm{C}$. IR $\left(v / \mathrm{cm}^{-1}\right): 3450-3250\left(2 \mathrm{NH}_{2}, \mathrm{NH}\right), 2980,2865\left(\mathrm{CH}_{3}, \mathrm{CH}_{2}\right), 2200$ $(\mathrm{CN}), 1730,1690(2 \mathrm{C}=\mathrm{O}) .{ }^{1} \mathrm{H}$ NMR $(\delta \mathrm{ppm}): 3.33\left(\mathrm{~s}, 3 \mathrm{H}, \mathrm{CH}_{3}\right), 3.43(\mathrm{~m}, 2 \mathrm{H}$, $\left.\mathrm{CH}_{2}\right), 3.70(\mathrm{~m}, 1 \mathrm{H}, \mathrm{CH}), 4.62\left(\mathrm{~s}, 4 \mathrm{H}, 2 \mathrm{NH}_{2}, \mathrm{D}_{2} \mathrm{O}\right.$-exchangeable $), 6.23(\mathrm{~s}, 1 \mathrm{H}$, $\mathrm{C}_{3}$-pyridine ring proton), $6.62(\mathrm{~s}, 1 \mathrm{H}, \mathrm{CH}), 7.00-7.32\left(\mathrm{~m}, 4 \mathrm{H}, \mathrm{C}_{6} \mathrm{H}_{4}\right), 10.87$ (s, $1 \mathrm{H}, \mathrm{NH}, \mathrm{D}_{2} \mathrm{O}$-exchangeable). $\mathrm{MS}(\mathrm{m} / \mathrm{z}, \%): 351\left(\mathrm{M}^{+}, 55 \%\right), 325\left(\mathrm{M}^{+}\right.$$\mathrm{CN}, 35 \%$ ), 130 (indolomethylene fragment, $\mathrm{C}_{9} \mathrm{H}_{8} \mathrm{~N}, 100 \%$ ). Anal. Calcd. for $\mathrm{C}_{18} \mathrm{H}_{17} \mathrm{~N}_{5} \mathrm{O}_{3}$ (351.36): $\mathrm{C}, 61.53 ; \mathrm{H}, 4.87 ; \mathrm{N}, 19.93$. Found: $\mathrm{C}, 61.21 ; \mathrm{H}, 4.73$; $\mathrm{N}, 19.67$.

Compound 6b: Pale yellow crystals, from $\mathrm{MeOH}$, yield $82 \%(1.44 \mathrm{~g})$, mp 198-199 ${ }^{\circ} \mathrm{C}$. IR $\left(v / \mathrm{cm}^{-1}\right): 3450-3250\left(\mathrm{NH}_{2}, \mathrm{NH}\right), 3050$ (CH-aromatic), 2225 $(\mathrm{CN}), 1730,1700(2 \mathrm{C}=\mathrm{O}), 1630(\mathrm{C}=\mathrm{C}) .{ }^{1} \mathrm{H}$ NMR $(\delta \mathrm{ppm}): 3.38\left(\mathrm{~s}, 3 \mathrm{H}, \mathrm{CH}_{3}\right)$, $3.43\left(\mathrm{~m}, 2 \mathrm{H}, \mathrm{CH}_{2}\right), 3.75(\mathrm{~m}, 1 \mathrm{H}, \mathrm{CH}), 4.41\left(\mathrm{~s}, 2 \mathrm{H}, \mathrm{NH}_{2}, \mathrm{D}_{2} \mathrm{O}\right.$-exchangeable $)$, $6.34\left(\mathrm{~s}, 1 \mathrm{H}, \mathrm{C}_{3}\right.$-pyridine ring proton), $6.82(\mathrm{~s}, 1 \mathrm{H}, \mathrm{CH}), 7.11-7.54(\mathrm{~m}, 4 \mathrm{H}$, $\left.\mathrm{C}_{6} \mathrm{H}_{4}\right), 10.05(\mathrm{~s}, 1 \mathrm{H}, \mathrm{OH})$. Anal. Calcd. for $\mathrm{C}_{18} \mathrm{H}_{16} \mathrm{~N}_{4} \mathrm{O}_{4}(352.35): \mathrm{C}, 61.35 ; \mathrm{H}$, 4.57; N, 15.90. Found: C, 61.19; H, 4.28; N, 15.73.

Methyl a-imino(5-aminopyrazolo-3-yl)-3-indolopropanoate (8a)

Methyl $\alpha$-imino(5-amino-2-phenylpyrazolo-3-yl)-3-indolopropanoate (8b)

General Procedure. To a solution, of compound 3 (1.42 g, $0.005 \mathrm{~mol})$ in ethanol $(30 \mathrm{~mL})$, containing a catalytic amount of triethylamine $(2 \mathrm{~mL}, 0.02$ $\mathrm{mol})$, equivalent amount of either hydrazine hydrate $(0.25 \mathrm{~g}, 0.005 \mathrm{~mol})$ or phenylhydrazine $(0.54 \mathrm{~g}, 0.005 \mathrm{~mol})$ was added. The reaction mixture in each case was heated under reflux for $5 \mathrm{~h}$ and then was cooled at room temperature, poured over ice/water mixture and neutralized with dilute hydrochloric acid. 
The formed solid product, in each case, was filtered off, washed with water and crystallized from the appropriate solvent.

Compound 8a: White crystals, from EtOH, yield 72\% (1.08 g), mp 154$155^{\circ} \mathrm{C}$. IR $\left(v / \mathrm{cm}^{-1}\right): 3450-3250\left(\mathrm{NH}_{2}, 3 \mathrm{NH}\right), 3050(\mathrm{CH}$-aromatic), 2985, 2865 $\left(\mathrm{CH}_{3}, \mathrm{CH}_{2}\right), 1720(\mathrm{C}=\mathrm{O}), 1660(\mathrm{C}=\mathrm{N}), 1620(\mathrm{C}=\mathrm{C}) .{ }^{1} \mathrm{H}$ NMR $(\delta \mathrm{ppm}): 3.35$ $\left(\mathrm{s}, 3 \mathrm{H}, \mathrm{CH}_{3}\right), 3.46\left(\mathrm{~m}, 2 \mathrm{H}, \mathrm{CH}_{2}\right), 3.73(\mathrm{~m}, 1 \mathrm{H}, \mathrm{CH}), 4.50\left(\mathrm{~s}, 2 \mathrm{H}, \mathrm{NH}_{2}, \mathrm{D}_{2} \mathrm{O}-\right.$ exchangeable), $5.83(\mathrm{~s}, 1 \mathrm{H}, \mathrm{CH}$ pyrazole $), 6.51(\mathrm{~s}, 1 \mathrm{H}, \mathrm{CH}), 6.98-7.40(\mathrm{~m}, 4 \mathrm{H}$, $\left.\mathrm{C}_{6} \mathrm{H}_{4}\right), 9.83,10.80,10.92\left(3 \mathrm{~s}, 3 \mathrm{H}, 3 \mathrm{NH}, \mathrm{D}_{2} \mathrm{O}\right.$-exchangeable). Anal. Calcd. for $\mathrm{C}_{15} \mathrm{H}_{17} \mathrm{~N}_{5} \mathrm{O}_{2}$ (299.33): C, 60.18, H, 5.72, N, 23.39. Found: C, 59.90; H, 5.43; N, 23.64 .

Compound 8b: Brown crystals, from EtOH, yield 75\% (1.40 g), mp. 90$91^{\circ} \mathrm{C}$. IR $\left(v / \mathrm{cm}^{-1}\right): 3400-3250\left(\mathrm{NH}_{2}, 2 \mathrm{NH}\right), 3050$ (CH-aromatic), 2985, 2865 $\left(\mathrm{CH}_{3}, \mathrm{CH}_{2}\right), 1730(\mathrm{C}=\mathrm{O}), 1660(\mathrm{C}=\mathrm{N}), 1620(\mathrm{C}=\mathrm{C}) .{ }^{1} \mathrm{H}$ NMR $(\delta \mathrm{ppm}): 3.40$ (s, $\left.3 \mathrm{H}, \mathrm{CH}_{3}\right), 3.51\left(\mathrm{~m}, 2 \mathrm{H}, \mathrm{CH}_{2}\right), 3.74(\mathrm{~m}, 1 \mathrm{H}, \mathrm{CH}), 4.23\left(\mathrm{~s}, 2 \mathrm{H}, \mathrm{NH}_{2}, \mathrm{D}_{2} \mathrm{O}-\right.$ exchangeable), $5.83(\mathrm{~s}, 1 \mathrm{H}, \mathrm{CH}$ pyrazole), $6.33(\mathrm{~s}, 1 \mathrm{H}, \mathrm{CH}), 7.32-7.55(\mathrm{~m}, 9 \mathrm{H}$, $\left.\mathrm{C}_{6} \mathrm{H}_{4}, \mathrm{C}_{6} \mathrm{H}_{5}\right), 10.80,10.92\left(2 \mathrm{~s}, 2 \mathrm{H}, 2 \mathrm{NH}, \mathrm{D}_{2} \mathrm{O}\right.$-exchangeable). MS (m/z, \%): $374\left(\mathrm{M}^{+}-1 ; 34 \%\right), 298\left(\mathrm{M}^{+}-\mathrm{C}_{6} \mathrm{H}_{5}, 57 \%\right), 77\left(\mathrm{C}_{6} \mathrm{H}_{5} ; 70 \%\right)$. Anal. Calcd. for $\mathrm{C}_{21} \mathrm{H}_{21} \mathrm{~N}_{5} \mathrm{O}_{2}$ (375.43): C, 67.18; H, 5.62; N, 18.65. Found: C, 67.17; H, 5.49; $\mathrm{N}, 18.52$.

3-Acetonitrilo-6-hydroxy-3-methylenoindolo-2,1,4 oxadiazine (9). To a solution of compound $3(1.42 \mathrm{~g}, 0.005 \mathrm{~mol})$ in ethanol $(30 \mathrm{~mL})$, containing a catalytic amount of sodium acetate $(1.0 \mathrm{~g})$ equivalent amount of hydroxylamine hydrochloric acid $(0.35 \mathrm{~g}, 0.005 \mathrm{~mol})$ in $\mathrm{H}_{2} \mathrm{O}(5 \mathrm{~mL})$ was added with stirring. The reaction mixture was stirred with warming for $30 \mathrm{~min}$, left to cool to $20{ }^{\circ} \mathrm{C}$. The formed solid product was triturated ice/water mixture, collected by filtration and crystallized from $\mathrm{MeOH}$. White crystals, yield $73 \%(0.97 \mathrm{~g})$, $\mathrm{mp} 94-95^{\circ} \mathrm{C}$. IR $\left(v / \mathrm{cm}^{-1}\right): 3300-3200(2 \mathrm{NH}, \mathrm{OH}), 3050(\mathrm{CH}$-aromatic), 2985 , $2865\left(\mathrm{CH}_{3}, \mathrm{CH}_{2}\right), 2200(\mathrm{CN}), 1660(\mathrm{C}=\mathrm{N}), 1620(\mathrm{C}=\mathrm{C}) .{ }^{1} \mathrm{H}$ NMR $(\delta \mathrm{ppm})$ : $1.25\left(\mathrm{~s}, 2 \mathrm{H}, \mathrm{CH}_{2}\right), 3.91\left(\mathrm{~s}, 2 \mathrm{H}, \mathrm{CH}_{2}\right), 6.64(\mathrm{~s}, 1 \mathrm{H}, \mathrm{CH}), 7.00-7.71\left(\mathrm{~m}, 4 \mathrm{H}, \mathrm{C}_{6} \mathrm{H}_{4}\right)$ 9.22, $9.52\left(2 \mathrm{~s}, 2 \mathrm{H}, 2 \mathrm{NH}, \mathrm{D}_{2} \mathrm{O}\right.$-exchangeable), $10.89(\mathrm{~s}, 1 \mathrm{H}, \mathrm{OH}) \cdot{ }^{13} \mathrm{C} \mathrm{NMR}(\mathrm{d}):$ 20.3, 21.6 (2CH2), 88.4 (oxadiazine C-4), 118.7, 119.4, 120.6, 120.9, 121.5, 130.4, 136.9 (benzene, pyrrole C), $118.7(\mathrm{CN}) .179 .8$ (oxadiazine C-3). Anal. Calcd. for $\mathrm{C}_{14} \mathrm{H}_{12} \mathrm{~N}_{4} \mathrm{O}_{2}$ (268.28). C, 62.68; H, 4.50; N, 20.83. Found: C, 62.59; $\mathrm{H}, 4.53 ; \mathrm{N}, 20.77$. (10a)

Methyl $\alpha$ - $N$-(5-amino-2,4-dicyanothiopheno-3-yl)-3-indolopropanoate

Methyl $\alpha-N$-(ethyl 5-amino-2-cyanothiopheno-3-yl-4-carboxylate)-3indolo-propanoate (10b)

\section{General Procedure}

To a mixture of compound 3 ( $1.42 \mathrm{~g}, 0.005 \mathrm{~mol})$, elemental sulfur $(0.16$ $\mathrm{g}, 0.005 \mathrm{~mol})$ and either malononitrile $4 \mathrm{a}(0.33 \mathrm{~g}, 0.005 \mathrm{~mol})$ or ethyl cyanoacetate $4 \mathbf{b}(0.56 \mathrm{~g}, 0.005 \mathrm{~mol})$ in ethanol $(30 \mathrm{~mL})$, triethylamine $(0.5 \mathrm{~mL}, 0.005$ mol) was added. The reaction mixture in each case was heated under reflux for $4 \mathrm{~h}$ and then was cooled at room temperature, poured over ice/water mixture and neutralized with dilute hydrochloric acid. The formed solid product was filtered off, dried and crystallized from the appropriate solvent.

Compound 10a: Brown crystals, from $\mathrm{MeOH}$, yield 73\% (1.33 g), mp 106$107^{\circ} \mathrm{C}$. IR $\left(v / \mathrm{cm}^{-1}\right): 3400-3250\left(\mathrm{NH}_{2}, 2 \mathrm{NH}\right), 3050(\mathrm{CH}$-aromatic), 2965, 2865 $\left(\mathrm{CH}_{3}, \mathrm{CH}_{2}\right), 2210,2225(2 \mathrm{CN}), 1730(\mathrm{C}=\mathrm{O}), 1620(\mathrm{C}=\mathrm{C}) .{ }^{1} \mathrm{H}$ NMR $(\delta \mathrm{ppm})$ : $3.39\left(\mathrm{~s}, 3 \mathrm{H}, \mathrm{CH}_{3}\right), 3.53\left(\mathrm{~m}, 2 \mathrm{H}, \mathrm{CH}_{2}\right), 3.75(\mathrm{~m}, 1 \mathrm{H}, \mathrm{CH}), 4.58\left(\mathrm{~s}, 2 \mathrm{H}, \mathrm{NH}_{2}, \mathrm{D}_{2} \mathrm{O}-\right.$ exchangeable), $6.53(\mathrm{~s}, 1 \mathrm{H}, \mathrm{CH}), 7.01-7.60\left(\mathrm{~m}, 4 \mathrm{H}, \mathrm{C}_{6} \mathrm{H}_{4}\right) 10.78,11.94(2 \mathrm{~s}, 2 \mathrm{H}$, $2 \mathrm{NH}, \mathrm{D}_{2} \mathrm{O}$-exchangeable). Anal. Calcd. for $\mathrm{C}_{18} \mathrm{H}_{15} \mathrm{~N}_{5} \mathrm{SO}_{2}(365.42)$ : C, 59.16; $\mathrm{H}$, 4.13; N, 19.16; S, 8.77. Found: C, 58.91; H, 4.05; N, 19.02; S, 8.51.

Compound 10b: Pale brown crystals, from EtOH, yield 75\% (1.54g), mp 91-92 ${ }^{\circ} \mathrm{C}$. IR $\left(v / \mathrm{cm}^{-1}\right): 3400-3250\left(\mathrm{NH}_{2}, 2 \mathrm{NH}\right) 3050(\mathrm{CH}$-aromatic), 2965, 2865 $\left(\mathrm{CH}_{3}, \mathrm{CH}_{2}\right), 2200(\mathrm{CN}), 1730,1700(2 \mathrm{C}=\mathrm{O}), 1620(\mathrm{C}=\mathrm{C}) .{ }^{1} \mathrm{H}$ NMR $(\delta \mathrm{ppm})$ : $1.23\left(\mathrm{t}, 3 \mathrm{H}, J=6.8 \mathrm{~Hz}\right.$, ester $\left.\mathrm{CH}_{3}\right), 3.41\left(\mathrm{~s}, 3 \mathrm{H}, \mathrm{CH}_{3}\right), 3.53\left(\mathrm{~m}, 2 \mathrm{H}, \mathrm{CH}_{2}\right), 3.75$ $(\mathrm{m}, 1 \mathrm{H}, \mathrm{CH}), 4.24\left(\mathrm{q}, 2 \mathrm{H}, J=6.8 \mathrm{~Hz}\right.$, ester $\left.\mathrm{CH}_{2}\right), 6.50\left(\mathrm{~s}, 2 \mathrm{H}, \mathrm{NH}_{2}, \mathrm{D}_{2} \mathrm{O}-\right.$ exchangeable), $6.99(\mathrm{~s}, 1 \mathrm{H}, \mathrm{CH}), 7.04-7.49\left(\mathrm{~m}, 4 \mathrm{H}, \mathrm{C}_{6} \mathrm{H}_{4}\right), 10.71,10.88(2 \mathrm{~s}$, $2 \mathrm{H}, 2 \mathrm{NH}, \mathrm{D}_{2} \mathrm{O}$-exchangeable). MS (m/z, \%): $412\left(\mathrm{M}^{+}, 62 \%\right)$. Anal. Calcd. for $\mathrm{C}_{20} \mathrm{H}_{20} \mathrm{~N}_{4} \mathrm{O}_{4} \mathrm{~S}$ (412.47): C, 58.23; H, 4.88; N, 13.58; S, 7.77. Found: C, 58.11; $\mathrm{H}, 4.72 ; \mathrm{N}, 13.43 ; \mathrm{S}, 7.62$.

Methyl $\alpha-N$-(phenylhydrazoacetonitrilocarbamido)-3 indolopropanoate (11). A solution of compound $3(1.42 \mathrm{~g}, 0.05 \mathrm{~mol})$, in ethanol $(30 \mathrm{~mL})$ containing a catalytic amount of sodium acetate $(1.0 \mathrm{~g})$ was cooled to $0-5^{\circ} \mathrm{C}$ and then treated gradually with a cold solution of benzenediazonium chloride (prepared from the appropriate quantities of aniline, $\mathrm{HCl}$ and $\mathrm{NaNO}_{2}$ ). After addition of the diazonium salt was completed, the reaction mixture was stirred at room temperature for $30 \mathrm{~min}$. The solid product, separated upon dilution with cold water, was filtered off, washed with water several times, dried and crystallized from EtOH to yield $72 \%(1.40 \mathrm{~g})$ pale brown crystals, $\mathrm{mp} 94-95^{\circ} \mathrm{C}$. IR $\left(v / \mathrm{cm}^{-1}\right): 3400,3300(3 \mathrm{NH}), 3050$ (CH-aromatic), 2985, $2865\left(\mathrm{CH}_{3}, \mathrm{CH}_{2}\right)$, $2200(\mathrm{CN}), 1720,1690(2 \mathrm{C}=\mathrm{O}), 1660(\mathrm{C}=\mathrm{N}), 1630(\mathrm{C}=\mathrm{C}) .{ }^{1} \mathrm{H}$ NMR $(\delta \mathrm{ppm})$ : $1.27(\mathrm{~s}, 1 \mathrm{H}, \mathrm{CH}), 3.37\left(\mathrm{~s}, 3 \mathrm{H}, \mathrm{CH}_{3}\right), 3.47\left(\mathrm{~m}, 2 \mathrm{H}, \mathrm{CH}_{2}\right), 3.70(\mathrm{~m}, 1 \mathrm{H}, \mathrm{CH}), 5.64$ $(\mathrm{s}, 1 \mathrm{H}, \mathrm{CH}), 7.67-7.74\left(\mathrm{~m}, 9 \mathrm{H}, \mathrm{C}_{6} \mathrm{H}_{4}, \mathrm{C}_{6} \mathrm{H}_{5}\right), 9.25,9.97-9.99(3 \mathrm{~s}, 3 \mathrm{H}, 3 \mathrm{NH}$, $\mathrm{D}_{2} \mathrm{O}$-exchangeable). Anal. Calcd. for $\mathrm{C}_{21} \mathrm{H}_{19} \mathrm{~N}_{5} \mathrm{O}_{3}$ (389.41): C, 64.77; H, 4.91; N, 17.98. Found: C, 64.67; H, 4.89; N, 17.88 .

Methyl $\alpha$ - $N$-(benzaloacetonitrilocarbamido)-3-indolopropanoate (12). To a solution of compound $3(1.42 \mathrm{~g}, 0.005 \mathrm{~mol})$ in ethanol $(30 \mathrm{~mL})$, containing triethylamine $(1 \mathrm{~mL}, 0.01 \mathrm{~mol})$, equivalent amount of benzaldehyde $(0.53$ $\mathrm{g}, 0.005 \mathrm{~mol}$ ) was added. The reaction mixture was heated under reflux for $3 \mathrm{~h}$. Then poured over ice/water mixture and neutralized with dilute hydrochloric acid. The formed solid product filtered off, dried and crystallized from EtOH. Pale brown crystals, yield $77 \%(1.43 \mathrm{~g})$, mp $81-82^{\circ} \mathrm{C}$. IR $\left(v / \mathrm{cm}^{-1}\right): 3400-3200$ $(2 \mathrm{NH}), 3050$ (CH-aromatic), 2985, $2865\left(\mathrm{CH}_{3}, \mathrm{CH}_{2}\right), 2200(\mathrm{CN}), 1720,1690$ $(2 \mathrm{C}=\mathrm{O}) .{ }^{1} \mathrm{H}$ NMR $(\delta \mathrm{ppm}): 3.40\left(\mathrm{~s}, 3 \mathrm{H}, \mathrm{CH}_{3}\right), 3.59\left(\mathrm{~m}, 2 \mathrm{H}, \mathrm{CH}_{2}\right), 3.72(\mathrm{~m}$, $1 \mathrm{H}, \mathrm{CH}), 4.10(\mathrm{~s}, 1 \mathrm{H}, \mathrm{CH}), 7.08(\mathrm{~s}, 1 \mathrm{H}, \mathrm{CH}), 7.31-7.72\left(\mathrm{~m}, 9 \mathrm{H}, \mathrm{C}_{6} \mathrm{H}_{4}, \mathrm{C}_{6} \mathrm{H}_{5}\right)$, $8.42,10.93\left(2 \mathrm{~s}, 2 \mathrm{H}, 2 \mathrm{NH}, \mathrm{D}_{2} \mathrm{O}\right.$-exchangeable). $\mathrm{MS}(\mathrm{m} / \mathrm{z}, \%): 374\left(\mathrm{M}^{+*}, 22 \%\right)$, $315\left(\mathrm{M}^{+}-\mathrm{COOCH}_{3}, 21 \%\right), 284\left(\mathrm{M}^{+}-\mathrm{CHPh}, 20 \%\right), 130$, (indolomethylene fragment, $\left.\mathrm{C}_{9} \mathrm{H}_{8} \mathrm{~N}, 100 \%\right)$. Anal. Calcd. for $\mathrm{C}_{22} \mathrm{H}_{10} \mathrm{~N}_{3} \mathrm{O}_{3}(373.41)$ : C, 70.76; $\mathrm{H}, 5.12$; $\mathrm{N}, 11.25$. Found: $\mathrm{C}, 70.55 ; \mathrm{H}, 5.11 ; \mathrm{N}, 11.13$.

Methyl $\alpha$-(6-amino-3,5-dicyano-2-oxo-4-phenylpyrido-1-yl) -3-indolopropanoate (14a)

Methyl $a$-(ethyl 6-amino-3-cyano-2-oxo-4-phenylpyrido-1-yl-5carboxylate)-3-indolopropanoate (14b)

General Procedure. To a solution of compound $12(1.86 \mathrm{~g}, 0.005 \mathrm{~mol})$ in ethanol $(30 \mathrm{~mL})$, containing $(1 \mathrm{~mL}, 0.01 \mathrm{~mol})$, equivalent amount of either malononitrile $4 \mathrm{a}(0.33 \mathrm{~g}, 0.005 \mathrm{~mol})$, or ethyl canoacetate $4 \mathrm{~b}(0.56 \mathrm{~g}, 0.005$ mol) was added. The reaction mixture, in each case, was heated under reflux for $3 \mathrm{~h}$ then poured over ice/water mixture and neutralized with hydrochloric acid. The formed solid product, in each case filtered off, dried, and crystallized from the appropriate solvent.

Compound 14a: yellow crystals, from EtOH, yield 72\% (1.58g), mp 118$120^{\circ} \mathrm{C}$. IR $\left(v / \mathrm{cm}^{-1}\right): 3450-3350\left(\mathrm{NH}_{2}\right), 3300,3200(2 \mathrm{NH}), 3050(\mathrm{CH}$-aromatic), 2985, $2865\left(\mathrm{CH}_{3}, \mathrm{CH}_{2}\right), 2220,2215(2 \mathrm{CN}), 1730,1690(2 \mathrm{C}=\mathrm{O}) .{ }^{1} \mathrm{H}$ NMR $(\delta \mathrm{ppm}): 3.39\left(\mathrm{~s}, 3 \mathrm{H}, \mathrm{CH}_{3}\right), 3.53\left(\mathrm{~m}, 2 \mathrm{H}, \mathrm{CH}_{2}\right), 3.75(\mathrm{~m}, 1 \mathrm{H}, \mathrm{CH}), 4.46(\mathrm{~s}, 2 \mathrm{H}$, $\mathrm{NH}_{2}, \mathrm{D}_{2} \mathrm{O}$-exchangeable $), 6.51,6.57(2 \mathrm{~d}, 2 \mathrm{H}, J=9.7 \mathrm{~Hz}, 2 \mathrm{CH}), 6.60(\mathrm{~s}, 1 \mathrm{H}$, $\mathrm{CH}), 7.02-7.60\left(\mathrm{~m}, 9 \mathrm{H}, \mathrm{C}_{6} \mathrm{H}_{5}, \mathrm{C}_{6} \mathrm{H}_{4}\right), 11.02\left(\mathrm{~s}, 1 \mathrm{H}, \mathrm{NH}, \mathrm{D}_{2} \mathrm{O}\right.$-exchangeable $)$.

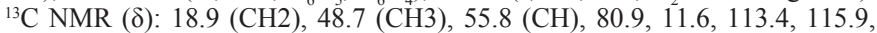
$118.4,120.1,122.7,130.5,133.8$ (benzene, pyrrole C), 118.6, $119.2(2 \mathrm{CN})$, 160.3, 170.4 (2CO). Anal. Calcd. for $\mathrm{C}_{25} \mathrm{H}_{21} \mathrm{~N}_{5} \mathrm{O}_{3}$ (439.37): C, 68.32; $\mathrm{H}, 4.81$; $\mathrm{N}, 15.93$. Found: $\mathrm{C}, 68.25 ; \mathrm{H}, 4.73 ; \mathrm{N}, 15.85$.

Compound 14b: White crystals, from dioxane, yield 77\% (1.87 g), mp $77-78^{\circ} \mathrm{C}$. IR $\left(v / \mathrm{cm}^{-1}\right): 3450-3250\left(\mathrm{NH}_{2}, \mathrm{NH}\right), 3050(\mathrm{CH}$-aromatic), 2985, 2865 $\left(\mathrm{CH}_{3}, \mathrm{CH}_{2}\right), 2200,(\mathrm{CN}), 1735,1730,1700(3 \mathrm{C}=\mathrm{O}), 1630(\mathrm{C}=\mathrm{C}),{ }^{1} \mathrm{H}$ NMR $(\delta$ ppm): 1.33 (t, $J=6.8 \mathrm{~Hz}, 3 \mathrm{H}$, ester $\left.\mathrm{CH}_{3}\right), 3.45\left(\mathrm{~s}, 3 \mathrm{H}, \mathrm{CH}_{3}\right), 3.52\left(\mathrm{~m}, 2 \mathrm{H}, \mathrm{CH}_{2}\right)$, $3.68(\mathrm{~m}, 1 \mathrm{H}, \mathrm{CH}), 4.32\left(\mathrm{q}, 2 \mathrm{H}, J=6.8 \mathrm{~Hz}\right.$, ester $\left.\mathrm{CH}_{2}\right), 5.18\left(\mathrm{~s}, 2 \mathrm{H}, \mathrm{NH}_{2}, \mathrm{D}_{2} \mathrm{O}-\right.$ exchangeable), 6.46, $6.52(2 \mathrm{~d}, 2 \mathrm{H}, J=9.7 \mathrm{~Hz}, 2 \mathrm{CH}), 6.73(\mathrm{~s}, 1 \mathrm{H}, \mathrm{CH}), 7.23-$ $7.92\left(\mathrm{~m}, 9 \mathrm{H}, \mathrm{C}_{6} \mathrm{H}_{5}, \mathrm{C}_{6} \mathrm{H}_{4}\right), 10.93$ (s, 1H, NH, $\mathrm{D}_{2} \mathrm{O}$-exchangeable). $\mathrm{MS}(\mathrm{m} / \mathrm{z}, \%)$ : $486\left(\mathrm{M}^{+}, 35 \%\right), 202\left[\mathrm{M}^{+}-\mathrm{C}_{15} \mathrm{H}_{14} \mathrm{~N}_{3} \mathrm{O}_{3}\right.$ (substituted pyridine fragment), 34\%], 130 (indolomethylene fragment, $\mathrm{C}_{9} \mathrm{H}_{8} \mathrm{~N}, 100 \%$ ). Anal. Calcd. for $\mathrm{C}_{27} \mathrm{H}_{26} \mathrm{~N}_{4} \mathrm{O}_{5}$ (486.53): C, 66.65; H, 5.38; N, 11.51. Found: C, 66.75; H, 5.28; N, 11.31 .

\footnotetext{
Methyl- $\alpha$-iminocarbonyl(3-amino-5-phenylpyrazolo-4-yl) -3-indolopropanoate (15a)

Methyl $\alpha$-iminocarbonyl(3-amino-1,5-diphenylpyrazolo-4-yl) $\quad-3$-indolopropanoate (15b)
}

\section{General Procedure}

To a solution of compound $12(1.86 \mathrm{~g}, 0.005 \mathrm{~mol})$ in ethanol $(30 \mathrm{~mL})$, containing triethylamine $(1 \mathrm{~mL}, 0.01 \mathrm{~mol})$, either hydrazine hydrate $(0.25 \mathrm{~g}$, $0.005 \mathrm{~mol})$ or phenylhydrazine $(0.54 \mathrm{~g}, 0.005 \mathrm{~mol})$ was added. The reaction mixture, in each case, was heated under reflux for $4 \mathrm{~h}$ then poured over ice/ water mixture and neutralized with hydrochloric acid. The formed solid prod- 
uct, in each case, was filtered off, washed with water and crystallized from the appropriate solvent.

Compound 15a: Yellow crystals, from EtOH, yield 77\% (1.55 g), mp 100$101^{\circ} \mathrm{C}$. IR $\left(v / \mathrm{cm}^{-1}\right): 3450-3150\left(\mathrm{NH}_{2}, 3 \mathrm{NH}\right), 3050(\mathrm{CH}$-aromatic), 2985, 2865 $\left(\mathrm{CH}_{3}, \mathrm{CH}_{2}\right), 1730,1700(2 \mathrm{C}=\mathrm{O}), 1660(\mathrm{C}=\mathrm{N}), 1630(\mathrm{C}=\mathrm{C}) .{ }^{1} \mathrm{H}$ NMR $(\delta \mathrm{ppm})$ : $3.43\left(\mathrm{~s}, 3 \mathrm{H}, \mathrm{CH}_{3}\right), 3.56\left(\mathrm{~m}, 2 \mathrm{H}, \mathrm{CH}_{2}\right), 3.75(\mathrm{~m}, 1 \mathrm{H}, \mathrm{CH}), 4.83\left(\mathrm{~s}, 2 \mathrm{H}, \mathrm{NH}_{2}, \mathrm{D}_{2} \mathrm{O}-\right.$ exchangeable), $6.93(\mathrm{~s}, 1 \mathrm{H}, \mathrm{CH}), 7.05-7.72\left(\mathrm{~m}, 9 \mathrm{H}, \mathrm{C}_{6} \mathrm{H}_{5}, \mathrm{C}_{6} \mathrm{H}_{4}\right), 9.48,10.91$, $10.92\left(3 \mathrm{~s}, 3 \mathrm{H}, 3 \mathrm{NH}, \mathrm{D}_{2} \mathrm{O}\right.$-exchangeable). $\mathrm{MS}(\mathrm{m} / \mathrm{z}, \%): 404\left(\mathrm{M}^{+}+1 ; 70 \%\right), 336$ $\left(\mathrm{M}^{+}-\mathrm{C}_{6} \mathrm{H}_{5}, 50 \%\right), 77^{2}\left(\mathrm{C}_{6} \mathrm{H}_{5} ; 36 \%\right)$. Anal. Calcd. for $\mathrm{C}_{22} \mathrm{H}_{21} \mathrm{~N}_{5} \mathrm{O}_{3}(403.44): \mathrm{C}$, 65.49; H, 5.24; N, 17.35. Found: C, 65.32; H, 5.11; N, 17.15 .

Compound 15b: White crystals from $\mathrm{MeOH}$, yield $72 \%(1.72 \mathrm{~g}), \mathrm{mp}$ 136-137 ${ }^{\circ} \mathrm{C}$. IR $\left(v / \mathrm{cm}^{-1}\right): 3450-3250\left(\mathrm{NH}_{2}, 2 \mathrm{NH}\right), 3050(\mathrm{CH}$-aromatic), 2985, $2685\left(\mathrm{CH}_{3}, \mathrm{CH}_{2}\right), 1730,1690(2 \mathrm{C}=\mathrm{O}), 1660(\mathrm{C}=\mathrm{N}), 1630(\mathrm{C}=\mathrm{C}) .{ }^{1} \mathrm{H}$ NMR $(\delta$ ppm): 3.43 (s, $\left.3 \mathrm{H}, \mathrm{CH}_{3}\right), 3.53\left(\mathrm{~m}, 2 \mathrm{H}, \mathrm{CH}_{2}\right), 3.75(\mathrm{~m}, 1 \mathrm{H}, \mathrm{CH}), 4.85(\mathrm{~s}, 2 \mathrm{H}$, $\mathrm{NH}_{2}, \mathrm{D}_{2} \mathrm{O}$-exchangeable $), 6.73(\mathrm{~s}, 1 \mathrm{H}, \mathrm{CH}), 6.76-7.80\left(\mathrm{~m}, 14 \mathrm{H}, 2 \mathrm{C}_{6} \mathrm{H}_{5}, \mathrm{C}_{\mathrm{H}}\right)$, 10.37, 10.93, (2s, 2H, 2NH, $\mathrm{D}_{2} \mathrm{O}$-exchangeable). Anal. Calcd. for $\mathrm{C}_{28} \mathrm{H}_{25} \mathrm{~N}_{5} \mathrm{O}_{3}$ (479.54): C, 70.13; H, 5.25; N, 14.60. Found: C, 69.99; H, 5.11; N, 14.42 .

Methyl $\alpha$-iminocarbonyl(3-amino-5-phenylisooxazolo-4-yl) -3-indolopropanoate (16). To a solution of compound $12(1.86 \mathrm{~g}, 0.005 \mathrm{~mol})$ in ethanol $(30 \mathrm{~mL})$, containing a catalytic amount of sodium acetate $(1.0 \mathrm{~g})$, equivalent amount of hydroxylamine hydrochloride acid $(0.35 \mathrm{~g}, 0.005 \mathrm{~mol})$ in ethanol $(10 \mathrm{~mL})$ was added with stirring and warming. The solid product formed at room temperature triturated with ice/water mixture and collected by filtration. Yellowish crystals, from $\mathrm{MeOH}$, yield $75 \%(1.51 \mathrm{~g}), \mathrm{mp} 136-137^{\circ} \mathrm{C}$. IR $(v /$ $\left.\mathrm{cm}^{-1}\right)$ : 3450-3250 $\left(\mathrm{NH}_{2}, 2 \mathrm{NH}\right), 3050$ (CH-aromatic), 2985, $2865\left(\mathrm{CH}_{3}, \mathrm{CH}_{2}\right)$, $1730,1690(2 \mathrm{C}=\mathrm{O}), 1660(\mathrm{C}=\mathrm{N}), 1630(\mathrm{C}=\mathrm{C}) .{ }^{1} \mathrm{H}$ NMR $(\delta \mathrm{ppm}): 3.38(\mathrm{~s}, 3 \mathrm{H}$, $\left.\mathrm{CH}_{3}\right), 3.55\left(\mathrm{~m}, 2 \mathrm{H}, \mathrm{CH}_{2}\right), 3.72(\mathrm{~m}, 1 \mathrm{H}, \mathrm{CH}), 4.83\left(\mathrm{~s}, 2 \mathrm{H}, \mathrm{NH}_{2}, \mathrm{D}_{2} \mathrm{O}\right.$-exchangeable), $6.31(\mathrm{~s}, 1 \mathrm{H}, \mathrm{CH}), 7.04-7.71\left(\mathrm{~m}, 9 \mathrm{H}, \mathrm{C}_{6} \mathrm{H}_{4}, \mathrm{C}_{6} \mathrm{H}_{5}\right), 10.91,10.97(2 \mathrm{~s}, 2 \mathrm{H}$, 2NH, $\mathrm{D}_{2} \mathrm{O}$-exchangeable). Anal. Calcd. for $\mathrm{C}_{22} \mathrm{H}_{20} \mathrm{~N}_{4} \mathrm{O}_{4}$ (404.43): C, 65.33; H, $4.98 ; \mathrm{N}, 13.85$. Found: C, 65.11; H, 4.72; N, 13.79.

$\alpha$-Imino(acetonitrilocarbamido)-3-indolopropionic acid (17). To a solution of tryptophan $\mathbf{1}(1.02 \mathrm{~g}, 0.005 \mathrm{~mol})$ in dimethyl formamide $(30 \mathrm{~mL})$, equimolar amount of ethyl cyanoacetate $4 \mathbf{b}(0.56 \mathrm{~g}, 0.005 \mathrm{~mol})$ was added. The reaction mixture was heated under reflux for $4 \mathrm{~h}$ and then was evaporated in vacuum. The remaining product was triturated with diethyl ether, and the solid product was collected by filtration. Yellow crystals, from EtOH, yield $79 \%$ $(1.07 \mathrm{~g}), \mathrm{mp} 146-147^{\circ} \mathrm{C}$. IR $\left(v / \mathrm{cm}^{-1}\right): 3430-3385(2 \mathrm{NH}), 3050$ (CH-aromatic), $2680\left(\mathrm{CH}_{2}\right), 2220(\mathrm{CN}), 1705,1680(2 \mathrm{C}=\mathrm{O}), 1620(\mathrm{C}=\mathrm{C}) .{ }^{1} \mathrm{H}$ NMR $(\delta \mathrm{ppm})$ : $3.43\left(\mathrm{~m}, 2 \mathrm{H}, \mathrm{CH}_{2}\right), 3.49\left(\mathrm{~s}, 2 \mathrm{H}, \mathrm{CH}_{2} \mathrm{CN}\right), 3.75(\mathrm{~m}, 1 \mathrm{H}, \mathrm{CH}), 6.95(\mathrm{~s}, 1 \mathrm{H}, \mathrm{CH})$, 7.01-7.49 (m, $\left.4 \mathrm{H}, \mathrm{C}_{6} \mathrm{H}_{4}\right), 8.75,8.82\left(2 \mathrm{~s}, 2 \mathrm{H}, 2 \mathrm{NH}, \mathrm{D}_{2} \mathrm{O}\right.$-exchangeable), 11.15 (s, $1 \mathrm{H}, \mathrm{OH})$. Anal. Calcd. for $\mathrm{C}_{14} \mathrm{H}_{13} \mathrm{~N}_{3} \mathrm{O}_{3}(271.28): \mathrm{C}, 61.98 ; \mathrm{H}, 4.83 ; \mathrm{N}, 15.48$. Found: C, $61.77 ; \mathrm{H}, 4.65 ; \mathrm{N}, 15.21$.

$\alpha$-Imino(benzaloacetonitriocarbamido)-3-indolopropionic acid (18). To a solution of compound $\mathbf{1 7}(1.35 \mathrm{~g}, 0.005 \mathrm{~mol})$, in ethanol $(30 \mathrm{~mL})$, containing piperidine $(0.85 \mathrm{~mL}, 0.01 \mathrm{~mol})$, equivalent amount of benzaldehyde $(0.53$ $\mathrm{g}, 0.005 \mathrm{~mol}$ ) was added. The reaction mixture was heated under reflux for $3 \mathrm{~h}$ then poured over ice/water mixture and neutralized with dilute hydrochloric acid. The formed solid product filtered off, dried and crystallized from dilute ethanol. Pale brown crystals, yield $75 \%(1.21 \mathrm{~g}), \mathrm{mp} 177-178^{\circ} \mathrm{C}$. IR $\left(\gamma / \mathrm{cm}^{-1}\right)$ : 3400-3200 (2NH), 3050 (CH-aromatic), $2865\left(\mathrm{CH}_{2}\right), 2200(\mathrm{CN}), 1720,1690$ $(2 \mathrm{C}=\mathrm{O}) .{ }^{1} \mathrm{H}$ NMR $(\delta \mathrm{ppm}): 3.53\left(\mathrm{~m}, 2 \mathrm{H}, \mathrm{CH}_{2}\right), 3.72(\mathrm{~m}, 1 \mathrm{H}, \mathrm{CH}), 4.10(\mathrm{~s}, 1 \mathrm{H}$, $\mathrm{CH}), 6.48(\mathrm{~s}, 1 \mathrm{H}, \mathrm{CH}), 7.31-7.72\left(\mathrm{~m}, 9 \mathrm{H}, \mathrm{C}_{6} \mathrm{H}_{4}, \mathrm{C}_{6} \mathrm{H}_{5}\right), 8.42,9.11(2 \mathrm{~s}, 2 \mathrm{H}, 2 \mathrm{NH}$, $\mathrm{D}_{2} \mathrm{O}$-exchangeable), 11.15 (s, $\left.1 \mathrm{H}, \mathrm{OH}\right) . \mathrm{MS}(\mathrm{m} / \mathrm{z}, \%): 359\left(\mathrm{M}^{+}, 35 \%\right)$. Anal. Calcd. for $\mathrm{C}_{21} \mathrm{H}_{17} \mathrm{~N}_{3} \mathrm{O}_{3}(359.38)$ : C, $70.18 ; \mathrm{H}, 4.76 ; \mathrm{N}, 11.69$. Found: $\mathrm{C}, 70.31$; $\mathrm{H}, 4.53 ; \mathrm{N}, 11.45$.

$\alpha$-Imino(phenylhydrazoacetonitrilocarbamido)-3-indolopropionic acid (19). A solution of compound $17(1.35 \mathrm{~g}, 0.005 \mathrm{~mol})$, in ethanol $(30 \mathrm{~mL})$, containing a catalytic amount of sodium acetate $(1.0 \mathrm{~g})$ was cooled to $0-5^{\circ} \mathrm{C}$ and then gradually with a cold solution of benzenediazonium chloride [prepared by adding sodium nitrite solution $(0.7 \mathrm{~g}, 0.01 \mathrm{~mol})$ to a cold solution of aniline $0.45 \mathrm{~g}, 0.005 \mathrm{~mol}$ ) with continuous stirring]. After addition of diazonium salt was completed, the reaction mixture was stirred at room temperature for $30 \mathrm{~min}$. The solid product, separated upon dilution with cold water, was filtered off, washed with water several times, dried and crystallized. Brown crystals from EtOH, yield $75 \%(1.40 \mathrm{~g})$, $\mathrm{mp} 79-80^{\circ} \mathrm{C}$. IR $\left(\gamma / \mathrm{cm}^{-1}\right): 3430-3335$ (3NH), 3050 (CH-aromatic), $2865\left(\mathrm{CH}_{2}\right), 2200(\mathrm{CN}), 1720,1690(2 \mathrm{C}=\mathrm{O})$, $1660(\mathrm{C}=\mathrm{N}), 1630(\mathrm{C}=\mathrm{C}) .{ }^{1} \mathrm{H}$ NMR $(\delta \mathrm{ppm}): 1.27(\mathrm{~s}, 1 \mathrm{H}, \mathrm{CH}), 3.28(\mathrm{~m}, 2 \mathrm{H}$,
$\left.\mathrm{CH}_{2}\right), 3.75(\mathrm{~m}, 1 \mathrm{H}, \mathrm{CH}), 5.64(\mathrm{~s}, 1 \mathrm{H}, \mathrm{CH}), 7.67-7.74\left(\mathrm{~m}, 9 \mathrm{H}, \mathrm{C}_{6} \mathrm{H}_{4}, \mathrm{C}_{6} \mathrm{H}_{5}\right), 8.51$, 9.32-9.36 (3s, 3H, 3NH, $\mathrm{D}_{2} \mathrm{O}$-exchangeable), $11.40(\mathrm{~s}, 1 \mathrm{H}, \mathrm{OH}) .{ }^{13} \mathrm{C} \mathrm{NMR}(\delta)$ : $25.0\left(\mathrm{CH}_{2}\right), 55.8(\mathrm{CH}), 80.7,111.6,113.6,114.8,118.6,120.4,122.2,130.4$, 133.9 (benzene, pyrrole $\mathrm{C}), 117.6(\mathrm{CN}), 150.3,166.4(2 \mathrm{CO}), 177.9(\mathrm{C}=\mathrm{N})$. Anal. Calcd. for $\mathrm{C}_{20} \mathrm{H}_{17} \mathrm{~N}_{5} \mathrm{O}_{3}$ (375.39): C, 63.99; $\mathrm{H}, 4.56 ; \mathrm{N}, 18.65$. Found: C, 63.78; H, 4.43; N, 18.59 .

\section{ACKNOWLEDGEMENT}

Mohareb R. M. expressed his deepest thank to the Alexander von Humboldt Foundation for financing support during his stay in Germany, Stuttgart University and completing this work.

\section{REFERENCES}

[1] T. R. Webb, C. Eigenbrot, J. Org. Chem. 56, 3009, (1991).

[2] C. H. Stammer, Tetrahedron 46, 2231, (1990).

[3] P. W. Schiller, G. Weltrowska, T. M. D. Nguyen, C. Lemieux, N. N. Chung, B. J. Marsden, B. C. Wilkes, J. Med. Chem., 34, 3125 (1991).

[4] G.M. Coppola, H.F. Schuster, Asymmetric Synthesis and Construction of Chiral Molecules Using Amino Acid, John Wiley\& Sons: New York, 1987.

[5] A. Baeyer, A. Emmerting, Ber., 2, 679 (1869).

[6] E. Fischer, F. Jourdan, Ber., 16, 2241 (1883).

[7] J. Bergman, E. Koch, B. Pelcman, Tetrahedron, 51, 5631 (1995).

[8] Ninomiya, Ichiya J. Nat. prod., 55, 541 (1992).

[9] B.A. Morgan, J. Singh, E. Baizman, H. Bently, D. Keifer, S. Ward, In Proceedings of the $10^{\text {th }}$ : American Peptide Symposium; Marshall G. R., Ed., Escom, Leiden, 1988, pp 508-509.

[10] J. Y. L. Chung, J. T. Wasicak, A. M. Nadzen, Synthetic Commun, 22, 1039 (1992).

[11] D. C. Horwell, P. D. Nichols, E. Roberts, Tetrahedron Lett., 35 (6), 939 (1994).

[12] A. Janczuk, W. Zhang, W. Xie, S. Lou, J. Cheng, P.G. Wang, Tetrahedron Lett., 43, 4271 (2002).

[13] V. S. Goodfellow, M. Settineri, R.G. Lawton, Bioochemistry, 28, 6346 (1989).

[14] T. Fujiwara, K. Omata, K. Kabuto, C. Kabuto, T. Takahashi, M. Segawa, Y. Takeuchi, Chem. Commun., 2694 (2001).

[15]S. H. Doss, R. M. Mohareb, G. A. Elmegeed, N. A. Louca, Pharmazie, 58, 607 (2003).

[16] S.H. Doss, N. A. Louca, G.A. Elmegeed, R.M. Mohareb, Arch. Pharm. Res., 22(5), 496 (1999).

[17] G.A. Elmegeed, H.H. Ahmed, J. S. Hussein, Eur. J. Med. Chem., 40, 1283 (2005).

[18] M.J. Queiroz, I.C. Ferreira, Y.D. Gaetano, G. Kirsch, R.C. Calhelha, L.M. Estevinho, Bioorg. Med. Chem., 14(20), 6827 (2006).

[19] R.G. Christiansen, M.R. Bell, T.E. D’Ambra, J.P. Mallamo, J.L. Herrmann, J.H. Ackerman, C. J. Opalka, R.K. Kullnig, R.C. Winneker, B.W. Snyder, F.H. Batzold, H.P. Schane, J. Med. Chem., 33, 2094 (1990). 